\section{Who are CT Dent?}

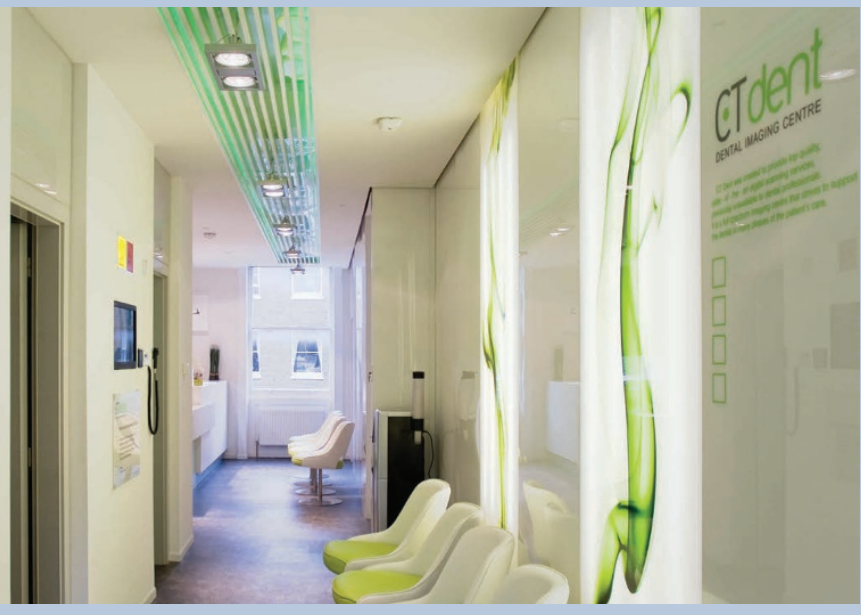

CT Dent was created to provide healthcare professionals with the best quality X-ray imaging using the latest state of the art technology. CT Dent launched its first imaging centre in the UK in 2007 in Devonshire Place, in the heart of central London. At the time cone beam computed tomography (CBCT) was still a relatively new imaging modality for dental and maxillofacial radiology, with the vast majority of CBCT scanners sited in dental hospitals. CT Dent saw there was a need for general dental practitioners to be able to refer their patients for this imaging to a specialist imaging centre. Since launching 11 years ago, CT Dent has grown significantly and now has over 7,000 registered users who refer their patients to six imaging centres in the UK. In addition to our imaging centres in London, Manchester, Birmingham, Bristol, Nottingham, and Leeds a seventh will soon be opening in Colchester, Essex.

Not only does CT Dent accept referrals for CBCT but also supports the dentist throughout the patient pathway with the many services offered. Our dedicated reformatting team process each X-ray dataset before it is returned to the dentist and our technical support and sales teams are always on hand to assist the dental team.

\section{Why choose CT Dent for your X-ray imaging}

As the technology for CBCT is continually evolving, CT Dent prides itself on having the latest X-ray technology. Our CBCT scanners have a variety of fields of view, resolutions and exposure factors which allow us to optimise your scan for all clinical needs, from implant prosthodontics, endodontics, orthodontics, oral medicine to ear nose and throat and maxillofacial reconstructive surgery.

All our X-ray imaging at CT Dent is taken by diagnostic radiographers who have previously worked in hospitals and have chosen to specialise in dental and maxillofacial radiography.

\section{How the process works?}

CT Dent makes it easy for healthcare professionals to use our services. Once they register their details they have access to our secure, web-hosted, management portal.

Each patient referral needs to be done in accordance with the Ionising Radiation (Medical Exposure) Regulations 2017 [IR(ME)R] and can be done electronically or on paper. The patient can either book an appointment or use our walk-in service. We can accommodate patients and return the results on the same day if the clinical need arises.

Once the radiographer has justified and completed the X-ray examination it takes on average 2-3 days to be returned to the referrer. It can be returned in a

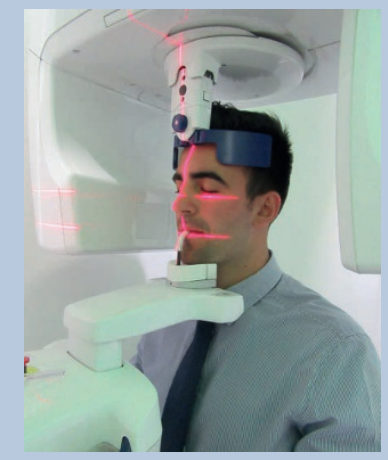
number of different formats including i-CAT Vision, CT Dent Picture Archiving and Communication System (PACS), Digital Imaging and Communications in Medicine (DICOM), SimPlant, MIS Mguide, NobelClinician, iDent and Planmeca Romexis. CT Dent also offers another formatting option. The X-ray dataset can be shown in a one-to-one ratio with volume rendered images on a PDF document or printed dye sublimation photopaper. Upon request CT Dent can also provide radiology reports written by a dental and maxillofacial radiologist.

\section{Educational opportunities}

CT Dent is committed to offering educational opportunities. Last year we launched our 'lunch and learn' sessions. These free sessions for registered clients are presented by our sales team and include lunch, provide demonstrations of our online booking system and the different formats we offer, as well as being an opportunity to discuss anything related with our service. These sessions have proven very popular with practices and they enhance the relationship we have with them.

In 2017 we also launched our book entitled Pathology, Anatomic Variants and Anomalies on CBCT scans. This has also proven to be very popular as a visual reference and learning tool for CBCT interpretation.

For 2018 we will be launching our e-learning platform.

If you would like to receive further information or have any questions about our services, please telephone 02074875717 email info@ct-dent.co.uk or write to us at CT Dent Ltd, Conan Doyle House, 2 Devonshire Place, London, W1G 6HJ.

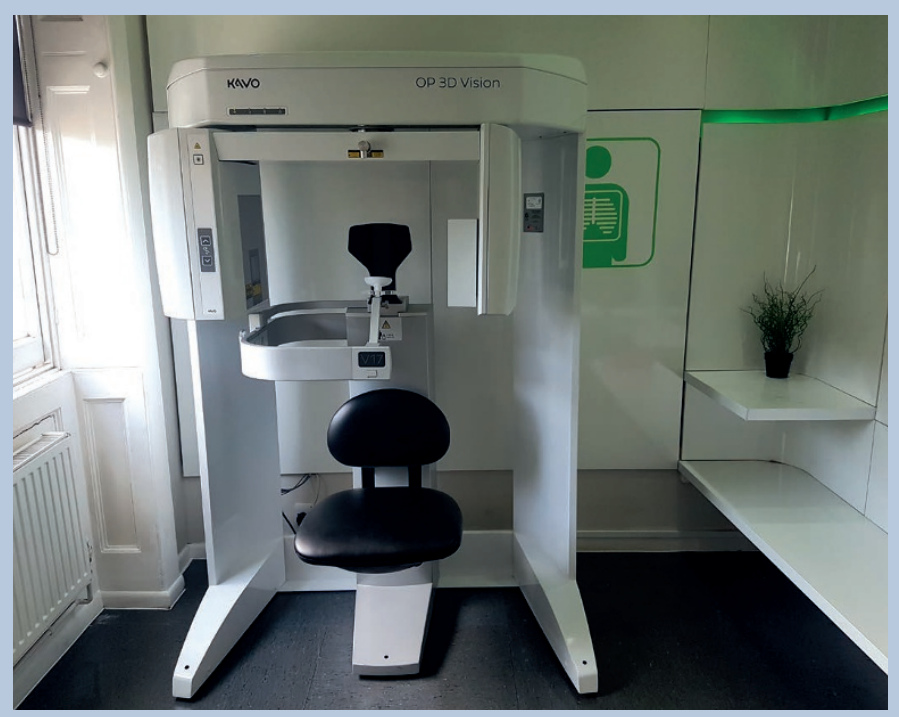

